\title{
Effects of Surgery and Asphyxia on Levels of Nucleosides, Purine Bases, and Lactate in Cerebrospinal Fluid of Fetal Lambs
}

\author{
HARMEN H. DE HAAN, ANKE C. M. IJZERMANS, JELTE DE HAAN, \\ HERMAN VAN BELLE, AND TOM H. M. HASAART \\ Department of Obstetrics and Gynecology, University Hospital, Maastricht, the Netherlands \\ [H.H.d.H., A.C.M.IJ., J.d.H., T.H.M.H.], and Department of Biochemistry, Janssen Research \\ Foundation, Beerse, Belgium [H.V.B.]
} \begin{abstract}
ABST
During severe oxygen shortage, the fetal brain resorts to
anaerobic metabolism and ATP becomes catabolized. High catabolites) in cerebrospinal fluid (CSF) may therefore be associated with increased neonatal neurologic morbidity. In 22 fetal lambs ( 3 to $5 \mathrm{~d}$ after surgery, gestational age $123.5 \pm 3.5 \mathrm{~d})$, arterial oxygen content was progressively reduced to $35 \%$ of the baseline value with a balloon occluder around the maternal common internal iliac artery. This resulted in a 1 -h period of asphyxia, leading to a $\mathrm{pH}$ of $7.02 \pm 0.03$ and a base excess of $-17.0 \pm 1.0 \mathrm{mM}$. Mortality was $50 \%$. CSF was sampled from the spinal cistern and analyzed using HPLC. During reoxygenation, hypoxanthine and xanthine may serve as substrate for xanthine oxidase with concomitant production of oxygen-derived free radicals, which may aggravate cerebral damage. The main difference between surviving and nonsurviving animals was the speed of increment of ATP catabolites in
\end{abstract}

CSF: in the surviving group levels increased steadily, recovery values being significantly elevated compared with asphyxia values, whereas in the nonsurviving group the rise was rapid and levels during asphyxia did not differ significantly from levels during recovery. We conclude that 1) catheterization of the spinal cistern leads to increased levels of CSF hypoxanthine, xanthine, and inosine, and 2) during fetal asphyxia, levels of these ATP catabolites and lactate in CSF increase. 3) Maximum levels are reached during the recovery period and are similar for surviving and nonsurviving animals, but during asphyxia CSF levels of hypoxanthine and lactate were higher in the nonsurviving fetuses. 4) The rate of increase of ATP catabolites in CSF is higher in the nonsurviving animals and may therefore be predictive for fetal death. (Pediatr Res 36: 595-600, 1994)

CSF, cerebrospinal fluid
Intrauterine oxygen shortage leads to anaerobic fetal metabolism. Due to an increase of cerebral blood flow (1) and preferential streaming of well-oxygenated blood in the heart (2), the fetal cerebrum is provided with as much oxygen as possible. During severe or sustained oxygen shortage, however, the brain too resorts to anaerobic metabolism. Lack of oxygen impairs mitochondrial functioning, leading to energy shortage (3); ATP becomes degraded to AMP and, after dephosphorylation, further to adenosine, inosine, and hypoxanthine. Serum levels of hypoxanthine are considered a sensitive indicator of the hypoxic insult (4). Moreover, formation of hypoxanthine and xanthine may aggravate outcome after such an insult, because during reoxygenation hypoxanthine and xan-

Received December 28, 1993; accepted May 9, 1994.

Correspondence: Harmen H. de Haan, M.D., Department of Obstetrics and Gynecology, University Hospital, P.O. Box 5800, 6202 AZ Maastricht, the Netherlands. thine may act as substrate for the enzyme xanthine oxidase, yielding oxygen-derived free radicals and subsequent damage in the newborn (5). This may explain bronchopulmonary dysplasia in the preterm infant, because levels of pulmonary free radical scavengers are low in preterm animals and reach peak values around term (6). Because the intestine is known to contain large amounts of xanthine oxidase (5) and the vitreous corpus of the eye contains large amounts of hypoxanthine, necrotizing enterocolitis and retinopathy of prematurity may well be a result of oxygen-derived free radical formation, too (4).

Regarding the brain, oxygen-derived free radical scavengers prevent the secondary cerebral hypoperfusion after asphyxia in neonatal lambs (7) and limit perinatal postasphyxial brain damage in rats (8). We measured levels of lactate, adenosine, inosine, guanosine, hypoxanthine, xanthine, and uric acid in CSF of fetal lambs during surgery and before, during, and after severe asphyxia induced 3 to $5 \mathrm{~d}$ after surgery. We hypothesized 
that the level of ATP catabolites, especially of hypoxanthine, in the fetal lamb brain may affect outcome after asphyxia due to the subsequent oxygen-derived free radical production. Therefore, levels of ATP catabolites in CSF of surviving fetuses were compared with those of nonsurviving animals.

\section{METHODS}

Surgery. Surgery was performed in 50 pregnant Dutch Texel sheep under general anesthesia. Gestational age varied between 110 and $128 \mathrm{~d}$ (mean $\pm \mathrm{SD}=119.9 \pm 2.7$ $\mathrm{d}$, term $=147 \mathrm{~d}$ ). Instrumentation, handling, and care of animals was performed as described previously (9). Briefly, a paramedian abdominal incision was made, and an inflatable balloon occluder was placed around the maternal common internal iliac artery. A hysterotomy was performed and the fetus partly extracted. A midline incision was made in the dorsal surface of the fetal neck, and the epidural cavity was opened (10). Epidural fat, containing small veins, was carefully swept away with a cotton-tipped applicator, and the spinal cavity was opened with a needle. The appearing CSF was sampled. A polyvinyl catheter (outer diameter $1.6 \mathrm{~mm}$, inner diameter $0.8 \mathrm{~mm}$ ) was inserted through the puncture hole into the spinal cavity and advanced 1.0 to $2.0 \mathrm{~cm}$ cranially until adequate CSF sampling was possible. The catheter was fixed with tissue glue and sutured to the fetal skin. The tip of the catheter was located in the spinal cistern. Furthermore, catheters were inserted in the fetal axillary and femoral arteries, the femoral vein, and the amniotic cavity. Electrodes to measure the fetal ECG and electrocorticogram were placed. Electrodes and catheters were exteriorized to the ewe's flank.

Measurements. The CSF catheter dead space was reduced to approximately $0.2 \mathrm{~mL}$, the volume obtained per sample was $\geq 0.6 \mathrm{~mL}$, including the dead space volume. All CSF samples (included the ones obtained during surgery) were withdrawn with a $1-\mathrm{mL}$ syringe, centrifuged ( $3 \mathrm{~min}$ at $13000 \mathrm{rpm}$ ), frozen in liquid nitrogen, and stored at $-73^{\circ} \mathrm{C}$. After storage, all samples were analyzed simultaneously. Concentrations of lactate were measured using an enzymatic procedure. Levels of nucleosides and bases were determined using HPLC (11).

Blood gas values and $\mathrm{pH}$ from the fetal aortic arch were measured with an automated analyzer (AVL, Radiometer, Copenhagen, Denmark) and corrected for $39^{\circ} \mathrm{C}$. $\mathrm{Hb}$ saturation was measured with an hemoximeter (OSM2 Hemoximeter, Radiometer). Arterial oxygen content was calculated as follows: arterial oxygen content $(\mathrm{mM})=\mathrm{Hb}$ concentration $(\mathrm{mM}) \times \mathrm{Hb}$ oxygen saturation $(\% / 100)$. During baseline, at the nadir of asphyxia, and twice in the recovery period, $2 \mathrm{~mL}$ of blood were withdrawn from the axillary artery and centrifuged $(3 \mathrm{~min}$ at $13000 \mathrm{rpm})$. Serum was frozen in liquid nitrogen and stored at $-73^{\circ} \mathrm{C}$ to determine lactate concentrations.

Experiments. Fifty fetuses were instrumented with a CSF catheter. In 15 animals, CSF sampling could only be performed one to four times. Because of this low frequency of sampling, changes due to the experiment might be missed; therefore, these fetuses were excluded, together with 13 preparations in which fetal death in the first $3 \mathrm{~d}$ after surgery occurred. Gestational age of the remaining 22 fetuses during experiments varied between 113 and $132 \mathrm{~d}$ (mean $\pm \mathrm{SD}=123.5 \pm 3.5 \mathrm{~d}$ ). Baseline values for fetal metabolic parameters were obtained during a period of $2 \mathrm{~h}$, in which fetal acid-base balance was analyzed every $15 \mathrm{~min}$.

Asphyxia was induced after 3 to $5 \mathrm{~d}$ of recovery from surgery by gradual reduction of maternal uterine blood flow with stepwise inflation of the balloon occluder around the common internal iliac artery. To maintain the fetus in a stable hemodynamic condition, uterine blood flow was gradually reduced over a period of 90 to $120 \mathrm{~min}$ until fetal arterial oxygen content reached approximately $35 \%$ of the baseline value. During this period, acidemia occurred. This latter asphyxial condition was maintained for $1 \mathrm{~h}$, referred to as severe asphyxia (time $=0$ to 60 $\min$ ). At time $=60 \mathrm{~min}$, the occluder was released and recovery monitored. The experiment was ended when either the fetus died or, in case of fetal survival, after $3 \mathrm{~d}$. When the fetus was still alive after $3 \mathrm{~d}$, a relaparotomy was performed under general anesthesia. The fetus was perfused in vivo with a fixative solution so histologic analysis of the brain could be performed (12).

Calculations and data analysis. Because CSF levels of lactate, nucleosides, and purine bases may be indicative for fetal survival after asphyxia, the 22 animals were divided into survivors and nonsurvivors. Survival was established if the fetus was still alive $12 \mathrm{~h}$ after asphyxia.

In both groups, mean baseline values (from time $=$ $-240 \mathrm{~min}$ to $-120 \mathrm{~min}$ ) were determined and compared with asphyxia values (from time $=0 \mathrm{~min}$ to time $=60$ $\min$ ) and with recovery values (from time $=60 \mathrm{~min}$ to time $=180 \mathrm{~min}$ ) using a Wilcoxon matched-pairs signedranks test.

Differences between survivors and nonsurvivors were tested with the two-tailed Mann-Whitney $U$ test. Within one animal, differences between parameters obtained at surgery and during baseline were tested with a paired $t$ test. For all statistical tests used, $p<0.05$ was accepted as the level of statistical significance.

\section{RESULTS}

Eleven of the 22 fetal lambs (50\%) survived more than $12 \mathrm{~h}$ after asphyxia. The limit of $12 \mathrm{~h}$ was arbitrarily chosen. Because only one animal survived in the range from 6 to $20 \mathrm{~h}$, a change of this limit would not result in major shifts from one group to another. The mean (median) \pm SD surviving time was $62.2(72) \pm 22 \mathrm{~h}$ and $2.4(2)$ $\pm 1.7 \mathrm{~h}$ for the surviving and the nonsurviving groups, respectively.

Figure 1 shows the time course of the metabolic condition of both groups. During occlusion, mean arterial oxygen content decreased, leading to anaerobic fetal 

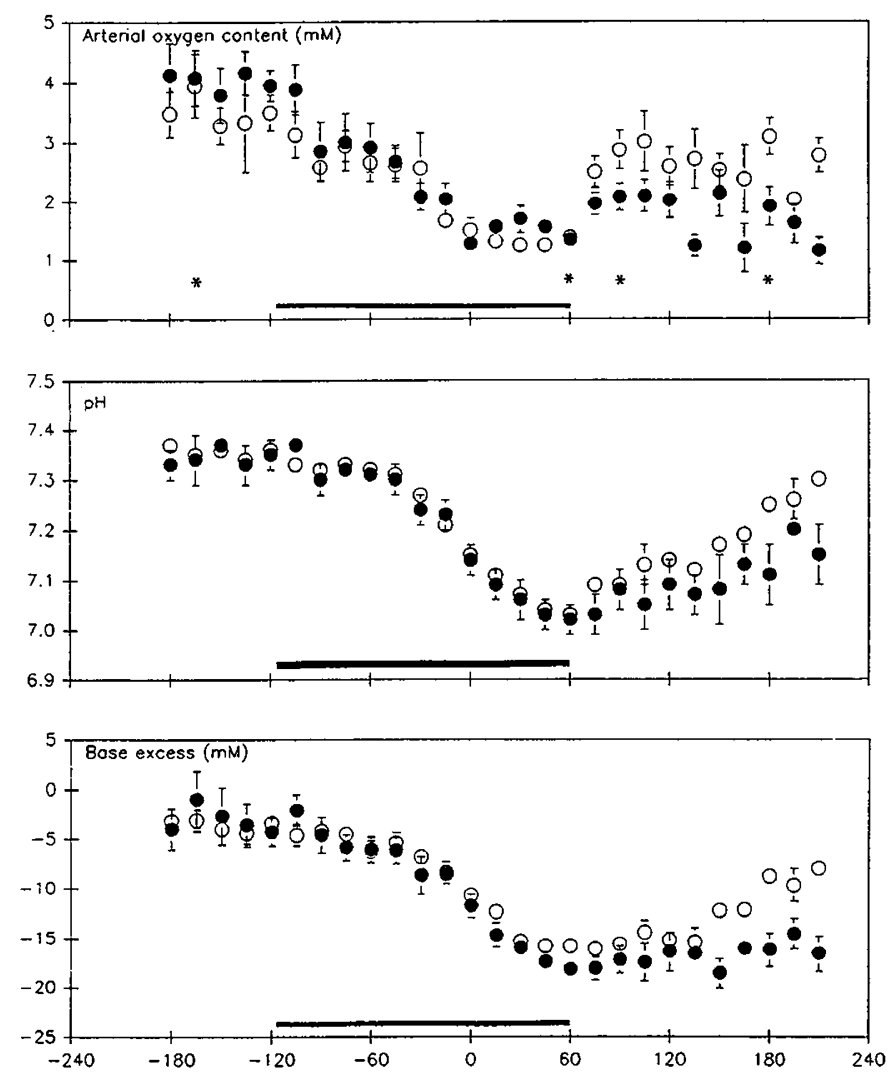

Figure 1. Time course of arterial oxygen content $(\mathrm{mM}), \mathrm{pH}$, and base excess $(\mathrm{mM})$. Values are means \pm SEM. Surviving animals $(n=11)$ are depicted as open circles, nonsurviving animals $(n=11)$ as filled circles. Time -180 to $-120 \mathrm{~min}=$ baseline period; time -120 to $0 \mathrm{~min}=$ progressive reduction of uterine blood flow; time 0 to $60 \mathrm{~min}=1-\mathrm{h}$ period of severe asphyxia; time 60 to $210 \mathrm{~min}=$ recovery period. Bar, period of occlusion. Asterisks in the upper panel represent serum lactate measurements.

metabolism and acidosis with a mean $\mathrm{pH}$ of $7.02 \pm 0.03$ at time $=60 \mathrm{~min}$. This was associated with a decrease of base excess to $-17.0 \pm 1.0 \mathrm{mM}$. After release of the occluder, the arterial oxygen content steadily increased in surviving animals, leading to $\mathrm{pH}$ and base excess values returning to baseline levels. This was in contrast with nonsurviving animals: release of the occluder resulted only temporarily in an increased arterial oxygen content, whereas $\mathrm{pH}$ and base excess did not return to baseline levels. No differences between groups were demonstrated before and during asphyxia, whereas from time $=150$ min onward arterial oxygen content, $\mathrm{pH}$, and base excess values were higher in the surviving group compared with the nonsurviving group $(p<0.05$; twotailed Mann-Whitney $U$ test).

Table 1 summarizes the effect of surgery on CSF lactate, hypoxanthine, xanthine, and inosine levels. Mean
Table 1. Effect of surgery on CSF lactate, hypoxanthine, xanthine, and inosine levels*

\begin{tabular}{lcccc}
\hline & $\begin{array}{c}\text { Lactate } \\
(\mathrm{mM})\end{array}$ & $\begin{array}{c}\text { Hypoxanthine } \\
(\mu \mathrm{M})\end{array}$ & $\begin{array}{c}\text { Xanthine } \\
(\mu \mathrm{M})\end{array}$ & $\begin{array}{c}\text { Inosine } \\
(\mu \mathrm{M})\end{array}$ \\
\hline Surgery & $2.59 \pm 0.31$ & $15.09 \pm 6.43$ & $5.85 \pm 1.86$ & $15.66 \pm 7.97$ \\
Baseline & $2.66 \pm 0.94$ & $9.80 \pm 2.83 \dagger$ & $4.08 \pm 2.46 \ddagger$ & $3.53 \pm 2.60 \dagger$ \\
\hline$*$ Values are depicted as mean $\pm \mathrm{SD} ; n=22$. \\
$\dagger p<0.001$ (surgery $v s$ baseline, paired $t$ test). \\
$\ddagger p<0.01$ (surgery vs baseline, paired $t$ test).
\end{tabular}

concentrations of these parameters at surgery were compared with mean values 3 to $5 \mathrm{~d}$ later at baseline measurement. For hypoxanthine, xanthine, and inosine, CSF concentrations during surgery were significantly elevated compared with baseline values in the experiment, whereas CSF lactate levels were not elevated during surgery. Values of adenosine, guanosine, and uric acid are not shown in Table 1 because these concentrations were not elevated due to surgery.

Serum lactate levels were measured at four moments in the experiment (Fig. 1). Results are shown in Table 2. During asphyxia, serum lactate levels increased 7- to 8 -fold, and a slow decrease of serum lactate levels was observed during recovery. Differences between survivors and nonsurvivors did not reach significance.

Due to sampling difficulties, withdrawal of CSF was not performed on standardized moments in the experiment. Besides the sample obtained during surgery, another five CSF samples were considered the minimum needed to be sure that changes induced by asphyxia were adequately measured. Figure 2 shows all individual CSF levels of lactate, hypoxanthine, and xanthine from surviving animals ( 11 animals, 73 samples, five to 10 samples per animal) and nonsurviving animals (11 animals, 75 samples, five to 11 samples per animal).

In Figure 3, CSF levels of adenosine, inosine, guanosine, and uric acid are depicted for surviving animals (11 animals, 73 samples, five to 10 samples per animal) and nonsurviving animals (11 animals, 75 samples, five to 11 samples per animal).

Statistical analysis of the scattergrams of Figures 2 and 3 is complicated by the different number of samples per animal obtained at unequal time points. Therefore, mean values of individual animals were calculated in the baseline period (from time $=-240 \mathrm{~min}$ to time $=-120 \mathrm{~min}$ ), in the period of asphyxia (from time $=0 \mathrm{~min}$ to time $=60$ $\mathrm{min}$ ), and in the recovery period (from time $=60 \mathrm{~min}$ to time $=180 \mathrm{~min})$. The means $\pm \mathrm{SD}$ of these values are presented for both surviving and nonsurviving groups in Table 3. During asphyxia and especially during recovery, levels were elevated compared with baseline values $(p<$

Table 2. Serum lactate levels $(m M)^{*}$

\begin{tabular}{|c|c|c|c|c|}
\hline & Baseline & $\begin{array}{c}\text { Asphyxia } \\
(\text { time }=60 \mathrm{~min})\end{array}$ & $\begin{array}{c}\text { Recovery } 1 \\
(\text { time }=90 \mathrm{~min})\end{array}$ & $\begin{array}{c}\text { Recovery } 2 \\
(\text { time }=180 \mathrm{~min})\end{array}$ \\
\hline Survivors & $1.33 \pm 0.46$ & $14.04 \pm 2.81$ & $13.27 \pm 2.47$ & $10.66 \pm 1.56$ \\
\hline Nonsurvivors & $1.86 \pm 0.73$ & $13.24 \pm 5.21$ & $12.68 \pm 5.00$ & $11.80 \pm 6.08$ \\
\hline
\end{tabular}

* Values are depicted as mean $\pm \mathrm{SD} ; n=11$ for both groups. No differences between groups. 

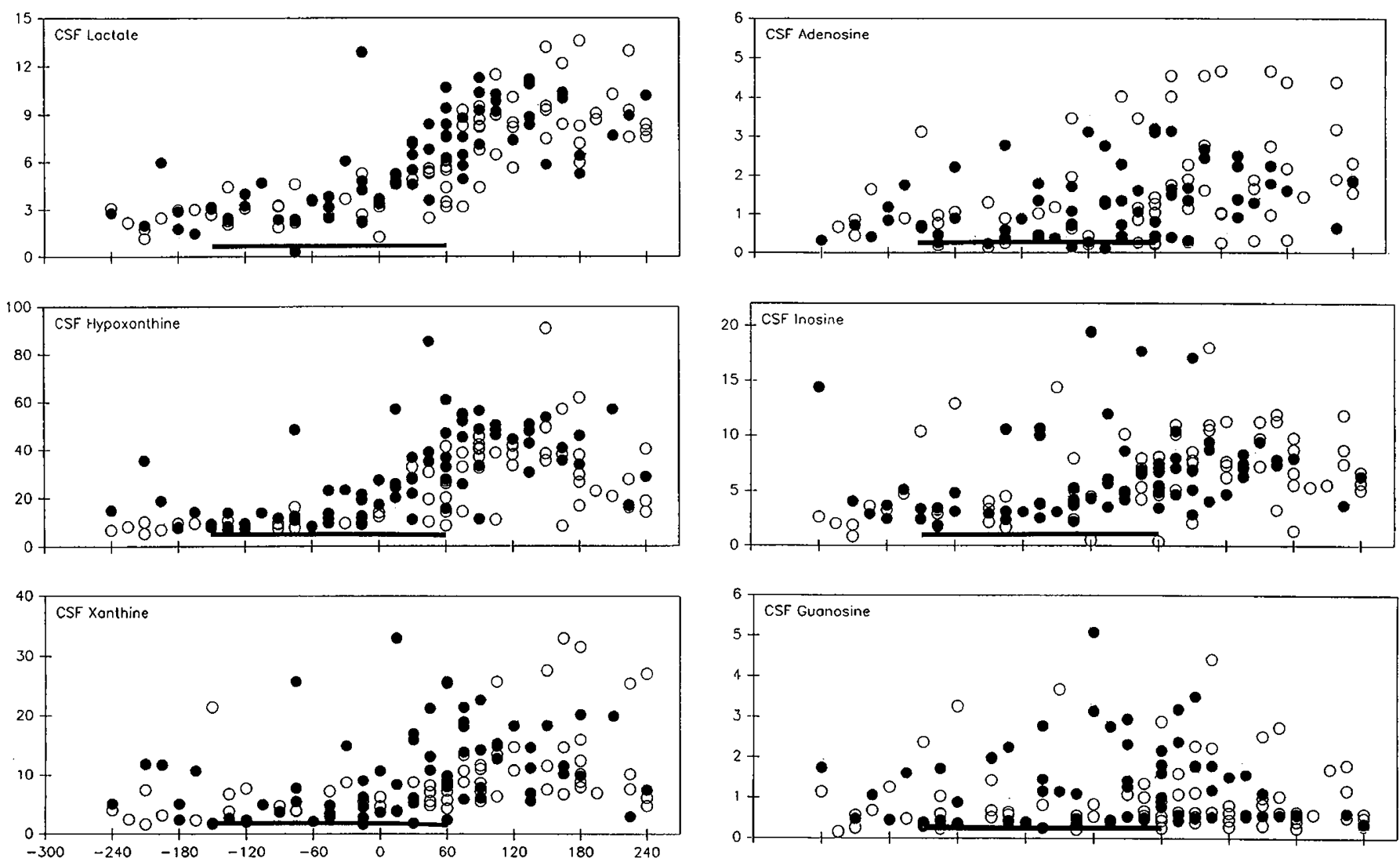

Figure 2. CSF levels of lactate (mM, upper panel), hypoxanthine ( $\mu \mathrm{M}$, middle panel), and xanthine ( $\mu \mathrm{M}$, lower panel). Time (min) is depicted on the $x$ axis. Time $=0 \mathrm{~min}$ to time $=60 \mathrm{~min}=1-\mathrm{h}$ period of severe asphyxia. All individual values are shown; open circles represent surviving fetuses, filled circles represent nonsurviving animals. Bar, period of occlusion.

0.05 and $p<0.01$, respectively; Wilcoxon matched-pairs signed-ranks test). During asphyxia, the mean levels of hypoxanthine and lactate in CSF were higher in the nonsurviving group compared with the surviving animals $(p<0.01$; two-tailed Mann-Whitney $U$ test). During recovery, the same levels were reached in both groups. The major difference between groups was the rate of increase of ATP catabolites in CSF. In the nonsurviving animals, the levels during recovery were not significantly higher than concentrations during asphyxia; in contrast, in the surviving fetuses the levels were elevated during asphyxia but increased even further during recovery $(p<$ 0.01; Wilcoxon matched-pairs signed-ranks test).

\section{DISCUSSION}

Purine nucleosides and bases (e.g. hypoxanthine, xanthine, inosine) are able to move freely between the inside and the outside of cells, whereas nucleotides (e.g. ATP, ADP, AMP) are larger and mainly confined in the cell interior. It is evident that extracellular levels of nucleosides and bases reflect the breakdown of intracellular nucleotides and are therefore indicative for cellular energy shortage or even for cellular damage (11). Because serum hypoxanthine levels during asphyxia mainly orig-

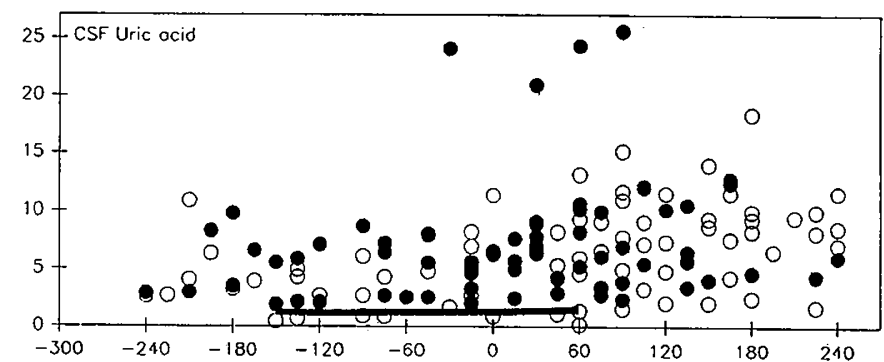

Figure 3. CSF levels of adenosine (first panel), inosine (second panel), guanosine (third panel), and uric acid (fourth panel). Values in $\mu \mathrm{M}$. Time $(\mathrm{min})$ is depicted on the $x$ axis. Time $=0 \mathrm{~min}$ to time $=60 \mathrm{~min}=$ 1-h period of severe asphyxia. All individual values are shown; open circles represent surviving fetuses, filled circles represent nonsurviving animals. Bar, period of occlusion.

inate from fetal heart and liver (13), we decided to determine nucleoside concentrations in CSF, especially because high levels of CSF hypoxanthine and xanthine after hypoxia have been associated with evidence of brain damage or subsequent death (14).

Table 1 stresses the importance of recovery after instrumentation. Complications during anesthesia can lead to oxygen shortage and ATP depletion, but in the present study CSF lactate levels were low, which is indicative for normal aerobic fetal metabolism. Surgery to expose the spinal cavity and the spinal tap were associated with cellular damage to such an extent that CSF hypoxanthine, xanthine, and especially inosine concentrations were significantly elevated compared with baseline levels 3 to $5 \mathrm{~d}$ after surgery. This trauma of instrumentation is in agreement with studies in rats in which placement of a 
Table 3. CSF levels of nucleosides, purine bases, and lactate during the experiment*

\begin{tabular}{|c|c|c|c|c|c|c|}
\hline & \multicolumn{3}{|c|}{ Survivors } & \multicolumn{3}{|c|}{ Nonsurvivors } \\
\hline & Baseline & Asphyxia & Recovery & Baseline & Asphyxia & Recovery \\
\hline Lactate $(\mathrm{mM})$ & $2.61 \pm 0.94$ & $4.49 \pm 1.29 \dagger \ddagger \S$ & $8.70 \pm 2.02 \dagger \ddagger$ & $3.04 \pm 1.14$ & $6.16 \pm 1.24 \dagger \ddagger \S$ & $9.23 \pm 1.83 \ddagger \|$ \\
\hline Hypoxanthine $(\mu \mathrm{M})$ & $8.52 \pm 1.84$ & $20.0 \pm 7.80 \ddagger \S \|$ & $38.1 \pm 13.7 \dagger \ddagger$ & $12.6 \pm 5.6$ & $33.3 \pm 10.9 \dagger \ddagger$ & $41.3 \pm 12.2 \|$ \\
\hline Xanthine $(\mu \mathrm{M})$ & $4.11 \pm 2.37$ & $6.80 \pm 1.33 \neq \|$ & $12.7 \pm 5.7 \dagger \neq$ & $5.06 \pm 3.18$ & $11.4 \pm 8.2 \dagger$ & $13.6 \pm 6.2 \|$ \\
\hline Adenosine $(\mu \mathrm{M})$ & $0.85 \pm 0.38$ & $1.48 \pm 1.25 \ddagger$ & $1.96 \pm 1.41 \dagger \ddagger$ & $0.95 \pm 0.68$ & $1.36 \pm 0.92$ & $1.60 \pm 0.89$ \\
\hline Inosine $(\mu \mathrm{M})$ & $3.70 \pm 3.42$ & $5.63 \pm 2.59 \ddagger$ & $8.10 \pm 2.55 \dagger \ddagger$ & $3.33 \pm 1.17$ & $6.97 \pm 3.15$ & $8.20 \pm 3.72 \|$ \\
\hline Guanosine $(\mu \mathrm{M})$ & $0.80 \pm 0.78$ & $1.07 \pm 0.72 \|$ & $1.14 \pm 0.87 \|$ & $0.86 \pm 0.63$ & $1.35 \pm 1.11$ & $1.46 \pm 1.00$ \\
\hline Uric acid $(\mu \mathrm{M})$ & $4.16 \pm 2.99$ & $6.16 \pm 3.72 \neq \|$ & $8.61 \pm 4.16 \dagger \ddagger$ & $5.70 \pm 2.38$ & $7.39 \pm 5.48$ & $8.08 \pm 7.13$ \\
\hline
\end{tabular}

${ }^{*}$ Values are depicted as mean $\pm \mathrm{SD} ; n=11$ for both groups. Baseline values are obtained during time $=-240$ to -120 min, asphyxia values from time $=0$ to $60 \mathrm{~min}$, and recovery values from time $=60$ to $180 \mathrm{~min}$.

$\dagger p<0.01$ (asphyxia or recovery $v s$ baseline, Wilcoxon matched-pairs signed-ranks test).

$\ddagger p<0.01$ (asphyxia $v s$ recovery, Wilcoxon matched-pairs signed-ranks test).

$\S p<0.01$ (survivors $v s$ nonsurvivors, two-tailed Mann-Whitney $U$ test).

$\| p<0.05$ (asphyxia or recovery $v s$ baseline, Wilcoxon matched-pairs signed-ranks test).

subdural catheter resulted in transient elevation of neuron-specific enolase levels in CSF (15). Because levels of CSF lactate, hypoxanthine, and xanthine at time $=60$ min were higher than during surgery, the asphyxial event will have induced more cellular damage than the trauma of surgery.

Oxygen shortage leads to catabolism of ATP, ADP, and AMP and to formation of adenosine. Adenosine is of great interest because it possesses strong biologic activity dependent on the 6-amino group (11). Adenosine causes coronary vasodilation and protects the myocardium after hypoxia-ischemia $(9,16)$. Removal of the 6-amino group of adenosine to form inosine is a fast process. Levels of CSF inosine increase during asphyxia and reach maximum values during recovery (Table 3 ). These peak values, however, were lower than levels obtained immediately after surgery (Table 1 ). We hypothesize that the difference observed is explained by the time needed for metabolism. Due to the short interval between the spinal tap and storage of CSF in liquid nitrogen during the surgical procedure, the metabolism of inosine to hypoxanthine has not yet happened. During asphyxia, however, the energy depletion has a more chronic character, and inosine is metabolized to hypoxanthine.

Values of hypoxanthine, xanthine, and inosine obtained in the current study are comparable with human data from newborns after hypoxia (14). A microdialysis technique showed similar hypoxanthine and xanthine levels in the extracellular compartment of the brain of severely asphyxiated exteriorized fetal lambs. These results, however, were obtained in anesthetized lambs with their heads fixed in a stereotactic frame (17). In the present study, CSF hypoxanthine levels were shown to rise during asphyxia. This is in agreement with the net cerebral efflux of hypoxanthine measured in plasma during severe asphyxia in fetal lambs (18). Maximum CSF hypoxanthine and CSF xanthine levels were reached in the hours after occlusion. The time lag between nadir of asphyxia and peak hypoxanthine and xanthine levels may be caused by damage to the blood-brain-CSF barrier after $1 \mathrm{~h}$ of severe asphyxia, by delayed neuronal damage (19), or by methodologic errors. Chances of the latter were small due to minimization of dead space of the sampling catheter, careful handling of samples, use of the recommended HPLC to determine nucleoside levels (11), and presence of this time lag in the majority of animals. The elevated level of CSF hypoxanthine and xanthine during the recovery period, in which availability of oxygen was almost normal, is considered important because both bases act as substrate for the enzyme xanthine oxidase in the production of oxygen-derived free radicals $(4,5,11)$. Xanthine oxidase is released during hypoxia from the fetal liver (4) and is present in the brain of fetal lambs (17). Indeed, asphyxiated fetal lambs with the highest CSF hypoxanthine and xanthine levels had the most extensive histologic brain damage (12). In this respect, the significantly $(p<0.01)$ higher levels of CSF hypoxanthine in nonsurviving animals compared with surviving fetuses during asphyxia may be of importance and may even predict fetal death. In the presence of elevated levels of cerebral hypoxanthine and xanthine, a large amount of oxygen administered during resuscitation after peripartal asphyxia may provide adverse effects. In newborn pigs, resuscitation after asphyxia with $100 \%$ oxygen was not shown to be beneficial compared with resuscitation with $21 \%$ oxygen (20). It may therefore be worthwhile to consider using $21 \%$ instead of $100 \%$ oxygen during resuscitation after peripartal asphyxia.

CSF lactate levels also reached maximum values during the recovery phase. Concentrations were lower than serum lactate levels (Table 2), which is in agreement with the net influx of lactate into the fetal lamb brain during severe asphyxia and recovery (18). Serum lactate measured during asphyxia originates from a variety of organs, such as, placenta, myocardium, and muscles, and is used in the brain as an energy source both during hypoxia (21) and during reoxygenation (18). The increase of CSF lactate in the recovery period is of less importance than CSF hypoxanthine and xanthine levels because involvement of lactate in oxygen-derived free radical production does not seem likely (5).

A remarkable difference between survivors and nonsurvivors is the rate of increase of ATP catabolites (and especially of hypoxanthine) in CSF. Whereas in the sur- 
viving group the levels at recovery were similar to the ones in nonsurvivors, the increment during asphyxia in nonsurvivors was twice the value in survivors. A similar tendency was observed for all parameters except guanosine. We have no clear explanation for this difference, nor do we know why it is most pronounced for hypoxanthine. It could be argued that during asphyxia in the nonsurvivors ATP catabolism proceeds much faster, but why does the difference persist during recovery? A second possibility may be the ability of the survivors to salvage hypoxanthine more efficiently. Third, it should be kept in mind that changes in CSF reflect an equilibrium between many processes, including formation and release from a variety of cells, uptake and catabolism in scavenging cells, and exchange with blood in the vasculature. Vascular efflux of ATP catabolites from CSF is guaranteed due to extensive fetal metabolism and placental diffusion. This implies that at a certain moment an equilibrium of maximal levels will be reached in CSF. This equilibrium may be reached during asphyxia in the nonsurviving animals, in contrast to the surviving fetuses, in which maximal levels are reached during recovery. The difference observed could be due to the larger amount of cellular damage in those fetuses that will not survive the asphyxial insult. Which one of the theories explains the difference between survivors and nonsurvivors cannot be decided from the present data. It may also be questioned whether the difference contributes to fetal death.

In summary, catheterization of the spinal cistern in preterm fetal lambs can be performed adequately and results in elevated CSF levels of inosine, hypoxanthine, and, to a lesser extent, xanthine. Experiments regarding CSF levels of nucleosides and purine bases should therefore preferably be performed in chronically instrumented animals. Three to $5 \mathrm{~d}$ after surgery, fetal anaerobic metabolism was initiated; CSF levels of lactate, hypoxanthine, and xanthine increased, whereas peak levels were reached in the recovery period. CSF hypoxanthine (and lactate) levels during asphyxia were higher in the nonsurviving animals. The level of hypoxanthine may be related to oxygen-derived free radical production, which aggravates brain damage. A second difference between groups was the rate of increase of the ATP catabolites in CSF, which was higher in the nonsurviving fetuses. Finally, CSF adenosine, guanosine, and uric acid seemed to increase in the survivors due to asphyxia, in contrast to the nonsurvivors.

\section{REFERENCES}

1. Peeters LLH, Sheldon RE, Jones MD, Makowski EL, Meschia G 1979 Blood flow to fetal organs as a function of arterial oxygen content. Am J Obstet Gynecol 135:637-646

2. Rudolph AM 1985 Distribution and regulation of blood flow in the fetal and neonatal lamb. Circ Res 57:811-821

3. Younkin DP, Wagerle LC, Chance B, Maria J, Delivoria-Papadopoulos M $1987^{31}$ P-NMR studies of cerebral metabolic changes during graded hypoxia in newborn lambs. J Appl Physiol 62:1569-1574

4. Saugstad OD 1988 Hypoxanthine as an indicator of hypoxia: its role in health and disease through free radical production. Pediatr Res 23:143-150

5. McCord JM 1985 Oxygen-derived free radicals in post-ischemic tissue injury. N Engl J Med 312:159-163

6. Tanswell AK, Freeman BA 1984 Pulmonary antioxidant enzyme maturation in the fetal and neonatal rat. I. Developmental profiles. Pediatr Res 18:584 587

7. Rosenberg AA, Murdaugh E, White CE 1989 The role of oxygen free radicals in postasphyxia cerebral hypoperfusion in newborn lambs. Pediatr Res 26:215-219

8. Thordstein M, Bågenholm R, Thiringer K, Kjellmer I 1993 Scavengers of free oxygen radicals in combination with magnesium ameliorate perinatal hypoxicischemic brain damage in the rat. Pediatr Res 34:23-26

9. de Haan HH, de Haan J, Van Reempts JLH, Van Belle H, Hasaart THM 1993 The effect of adenosine transport inhibition on cardiovascular function and survival after severe asphyxia in fetal lambs. Pediatr Res 33:185-189

10. Bissonnette JM, Hohimer AR, Richardson BS 1981 Ventriculocisternal cerebrospinal perfusion in unanesthetized fetal lambs. J Appl Physiol 50:880-883

11. Harkness RA 1988 Hypoxanthine, xanthine and uridine in body fluids, indicators of ATP depletion. J Chromatogr 429:255-278

12. de Haan HH, Van Reempts JLH, Vles JSH, de Haan J, Hasaart THM 1993 Effects of asphyxia on the fetal lamb brain. Am J Obstet Gynecol 169:1493 1501

13. Thiringer K, Karlsson K, Rosen KG, Kjellmer I 1984 Contribution of heart muscle, liver, skeletal muscle and placenta to the asphyxial hypoxanthine elevation in the acutely exteriorised fetal lamb. Biol Neonate 45:169-182

14. Harkness RA, Lund RJ 1983 Cerebrospinal fluid concentrations of hypoxanthine, xanthine, uridine and inosine: high concentrations of the ATP metabolite, hypoxanthine, after hypoxia. J Clin Pathol 36:1-8

15. Hårdemark HG, Persson L, Bolander HG, Hillered L, Olsson Y, Påhlman S 1988 Neuron-specific enolase is a marker of cerebral ischemia and infarct size in rat cerebrospinal fluid. Stroke 19:1140-1144

16. Van Belle H 1993 Nucleoside transport inhibition: a therapeutic approach to cardioprotection via adenosine? Cardiovasc Res 27:68-76

17. Kjellmer I, Andiné P, Hagberg H, Thiringer K 1989 Extracellular increase of hypoxanthine and xanthine in the cortex and basal ganglia of fetal lambs during hypoxia-ischemia. Brain Res 478:241-247

18. Thiringer K, Blomstrand S, Hrbek A, Karlsson K, Kjellmer I 1982 Cerebral arterio-venous difference for hypoxanthine and lactate during graded asphyxia in the fetal lamb. Brain Res 239:107-117

19. Pulsinelli WA, Brierley JB, Plum JB 1982 Temporal profile of neuronal damage in a model of transient forebrain ischemia. Ann Neurol 11:491-498

20. Rootwelt T, Loberg L, Moen A, Oyasæter S, Saugstad OD 1992 Hypoxemia and reoxygenation with $21 \%$ or $100 \%$ oxygen in newborn pigs: changes in blood pressure, base deficit, and hypoxanthine and brain morphology. Pediatr Res 32:107-113

21. Mann LL 1970 Effect of hypoxia on fetal cephalic blood flow, cephalic metabolism and the electroencephalogram. Exp Neurol 29:336-348 